respondents, 98\% reported asking pts about adherence to RA medication, even though $61 \%$ felt there was no way of knowing if pts were taking medications as prescribed and, overall, HCPs responded that $64 \%$ of pts did not always take RA medication as prescribed. While $87 \%$ of HCPs agreed that bDMARDs were most effective when taken in combination with csDMARDs and $92 \%$ reiterated this to pts, $62 \%$ were concerned that pts take bDMARDs without csDMARDs. Pts and caregivers reported that pts often stopped taking csDMARDs; however, HCPs underestimated the number of non-adherent pts (Figure A). The most common reason given by pts and caregivers for non-adherence to RA medication was difficulty in remembering to take medication; however, concerns about feeling ill and potential side effects were also common (Figure B). Despite this, $47 \%$ of pts and $51 \%$ of caregivers responded that pts often don't share all side effects caused by their RA medication with HCPs, while $38 \%$ of HCPs believed that pts did not inform them of all side effects.

Conclusion: This study from a large, multinational cohort of pts with RA, caregivers and HCPs describes medication adherence patterns. Pts on bDMARDs often did not take their background csDMARDs; however, the extent of pt non-adherence was underestimated by HCPs. These results highlight gaps in communication and understanding of non-adherence between pts, caregivers and HCPs.

Acknowledgement: Study sponsored by Pfizer Inc. Medical writing support was provided by Anthony G McCluskey, PhD, of CMC Connect and funded by Pfizer Inc. Disclosure of Interests: James Galloway Consultant for: Pfizer Inc, Ara Dikranian Consultant for: AbbVie, Pfizer Inc, Speakers bureau: AbbVie, Pfizer Inc, Cheryl L Koehn Shareholder of: Arthritis Consumer Experts, Grant/research support from: Canadian Institutes of Health Research, Arthritis Research Canada, St. Paul's Hospital, Employee of: Arthritis Consumer Experts, David C Gruben Shareholder of: Pfizer Inc, Employee of: Pfizer Inc, John Woolcott Shareholder of: Pfizer Inc, Employee of: Pfizer Inc, Sander Strengholt Shareholder of: Pfizer Inc, Employee of: Pfizer Inc DOI: 10.1136/annrheumdis-2019-eular.761

\section{AB0248 DISEASE ACTIVITY IS ASSOCIATED WITH FATIGUE IN THE FOLLOW-UP OF PATIENTS WITH RHEUMATOID ARTHRITIS}

Rocío Violeta Gamboa Cárdenas ${ }^{1}$, Manuel F. Ugarte-Gil ${ }^{2}$, Cristina

Reategui Sokolova ${ }^{3}$, Luz Hipólito ${ }^{4}$, Mariela Medina ${ }^{3}$, Claudia Elera-Fitzcarrald ${ }^{5}$ Victor Pimentel Quiroz ${ }^{3}$, Paola Alejandra Zeña Huancas ${ }^{3}$, Francisco Zevallos ${ }^{3}$, Samira Garcia-Hirsh ${ }^{3}$, Luciana Gil ${ }^{3}$, Zoila Rodriguez Bellido ${ }^{3}$, Cesar

Pastor Asurza ${ }^{3}$, Risto Perich Campos ${ }^{3} .{ }^{1}$ Hospital nacional Guillermo Almenara es Salud, Rheumatology, Lima, Peru; ${ }^{2}$ Universidad Científica del Sur, Rheumatology, Lima, Peru; ${ }^{3}$ Hospital Nacional Guillermo Almenara Essalud, Rheumatology, Lima, Peru; ${ }^{4}$ Hospital Emergencias Grau EsSalud, Rheumatology, Lima, Peru;

${ }^{2}$ Universidad Científica del Sur, Rheumatology, Lima, Peru

Background: Chronic fatigue negatively impacts on quality of life in Rheumatoid Arthritis (RA) patients $(1,2)$, does not allow optimal clinical results, being an negative predictor for clinical remission $(3,4)$.

Objectives: The objective was to determine whether disease activity correlates with increased fatigue in the follow-up of a RA patients.

Methods: Prospective study of the RA-Almenara cohort (criteria ACR87/ ACR-EULAR2010). Patients were included with at least two evaluations (half-yearly). Patients with fibromyalgia, major depression and/or anxious depressive disorder, chronic neuropathic pain or active systemic manifestations (including interstitial lung disease) were excluded. Disease activity was evaluated with SDAI (Simple Disease Activity Index) and fatigue with FACIT questionnaire (Functional Assessment of Chronic Illness Therapyfatigue). A generalized estimation equation model was used to determine the association between SDAI and FACIT, at each visit according with two models of analyses. Model 1 considered SDAI value as a linear variable; Model 2 considered each category of SDAl (high, moderate and low) activity, using remission as reference. Multivariable analyses were adjusted by possible confounders: gender, age at diagnosis, instruction, socio-economic level (Graffar), disease duration, tobacco, ACPA level, disability (MDHAQ), use of conventional (c) and biologic(b) DMARDs and corticosteroids (current use, past or non-use).

Results: Four hundred and twenty patients were included, 372 (88.6\%) women, age at diagnosis was 44.1 (13.2) years, disease duration 17.7 (11.6) years. At the baseline $4.2 \% 13.6 \%, 41.0 \%$ and $41.2 \%$ were in remission, low, moderate and high activity respectively; $40 \%$ were using corticosteroids, $256(61 \%)$ cDMARDs and $25(6 \%)$ bDMARDs. Basal SDAl was 23.7 (20.4) and FACIT 17.2 (8.8). We analyzed 1233 follow up visits [2.2 (1.2) visits per patient]. Multivariablee analysis: In model 1 were associated with FACIT: SDAI [B: -0.03 (IC95\%: - 0.06- $-0.01 ; \mathrm{p}=0.013$ ] and MHAQ [B: -9.55 (IC95\%: - 10.55- -0.54), $\mathrm{p}=<0.001]$. In model 2, were associated with FACIT: the categories high [B: -4.92 (IC95\%: -6.87 - 2.7); $p<0.001]$, moderate [B: -3.75 (IC95\% : -5.70- -1.89), $p<0.001]$ and low [B: $-2.22(95 \% \mathrm{Cl}$ : - 4.05- -0.39$) ; p=0.018]$ disease activity by SDAl, in addition with MHAQ [B: -9.11 (IC95\%: - 10.11- -8.11), $p<0.001]$ and tobacco use [B: $-3.37(95 \% \mathrm{Cl}$ - $6.56--0.18, \mathrm{p}=0.039)$ ]

Conclusions: Higher disease activity correlates with worse fatigue scores, at each follow-up visits of patients with RA

\section{REFERENCE:}

[1] Balsamo. Isr. Med. Assoc. J. 2014, 16, 57-60. 2. Alomari. Sci. World J. 2012:580863. doi: 10.1100/2012/580863. 3. Roodenrijs. Ann Rheum Dis. 2018 Sep 7. pii: annrheumdis-2018-213687. 4 Uhlig. J Rheumatol. 2016;43(4):716-23

Disclosure of Interests: None declared DOI: 10.1136/annrheumdis-2019-eular.5625

\section{AB0249 EFFECT OF RF AND ACPA NEGATIVATION IN CLINICAL RESPONSE IN RA PATIENTS UNDER BIOLOGIC THERAPY}

Sara Ganhão ${ }^{1}$, Raquel Ferreira ${ }^{1}$, Bruno Miguel Fernandes ${ }^{1}$, Salomé Garcia ${ }^{1}$ Eva Mariz ${ }^{1}$, Miguel Bernardes ${ }^{1,2}$, Lúcia Costa ${ }^{1}{ }^{1}$ Centro Hospitalar e Universitário de São João, Department of Rheumatology, Oporto, Portugal; ${ }^{2}$ Faculty of Medicine of Oporto University, Oporto, Portugal

Background: Both rheumatoid factor (RF) and antibodies against cyclic citrullinated peptide (ACPA) are regarded as serological markers of Rheumatoid Arthritis (RA), being well recognized as diagnostic and prognostic tools. However, their potential role in the disease's monitoring and clinical response is still under debate.

Objectives: To assess the effect of RF and ACPA negativation in clinical response to biologic therapy in RA patients.

Methods: Longitudinal retrospective study of RA patients treated with biologic therapy as first line option. Demographic and clinical data were collected at baseline and at 24 months follow-up, including: RF and ACPA status (negative or positive), ESR, CRP, DAS28 4v ESR, CDAl, SDAI, $H A Q$, EULAR and ACR response. RF was considered positive if $\geq 30$ $\mathrm{U} / \mathrm{ml}$ and ACPA if $>7 \mathrm{U} / \mathrm{ml}$. SPSS statistics 22.0 was used for statistical analysis.

Results: 169 patients were included with mean $( \pm S D)$ age of $50.7 \pm$ 10.6 years and median disease duration (min-max) of $10.2(0.69-39.4)$ years. The majority were female $(84 \%)$. At baseline $160(94.7 \%)$ were positive for RF and 166 (98.2\%) were positive for ACPA. 77 (45.6\%) turned negative for RF and/or ACPA at a median time of 20.3 months after biologic therapy begining. 63 out of 169 patients become negative for RF (37.3\%) and 17 patients for ACPA (10.1\%). 52 (30.6\%) patients were treated with etanercept, $39(22.9 \%)$ adalimumab, $27(15.9 \%)$ rituximab, 19 (11.2\%) tocilizumab; 16 infliximab (9.4\%); 14 (8.2\%) golimumab; $1(0.6 \%)$ certolizumab and $1(0.6 \%)$ anakinra. The mechanism of action of the drug didn't differ between patients who became seronegative for RF and/or ACPA and those who remained seropositive $(70.2 \%$ under anti-TNF $\alpha$ agents vs $73.9 \%$ for the other biologics). Most of the patients in the first group began adalimumab (32.5\%) and most of the patients in the latter began etanercept (34.8\%). Demographic characteristics like age, sex, disease duration, and extraarticular manifestations were comparable in both groups. They weren't comparable in terms of smoking habits $(p=0.014)$ : just $3(3.9 \%)$ current smokers in those who became negative for RF and/or ACPA vs 17 (18.5\%) among those who remained seropositive. Nevertheless, there weren't statistically significant differences in $\triangle \mathrm{DAS} 284 \mathrm{v}$ ESR, $\triangle \mathrm{ERS}, \triangle \mathrm{CRP}, \triangle \mathrm{CDAl}, \triangle \mathrm{SDAl}, \triangle \mathrm{HAQ}$, EULAR or ACR response at 24-months between patients with negativation of at least one antibody and those who remained seropositive.

Conclusion: In our sample, change of antibody status wasn't predictor of better response to biologic therapy. Therefore the results did not support the association between the persistence of RF or ACPA and the lack of effectiveness of biologic therapy at 2 years of treatment, but further studies are needed.

\section{REFERENCE:}

[1] Alexandra B. et al. Prospective cohort study of effects of infliximab on rheumatoid factor, anti-cyclic citrullinated peptide antibodies and antinuclear antibodies in patients with long-standing rheumatoid arthritis; Joint Bone Spine 76 (2009) 248e253.

Disclosure of Interests: None declared

DOI: 10.1136/annrheumdis-2019-eular.5294 